\title{
Calibration Of INERTIAL SENSOR By USING Particle Swarm Optimization ANd Human OPINION DYNAMICS AlgorithM
}

\author{
Vikas Kumar Sinha ${ }^{1}$, Avinash Kumar Maurya ${ }^{2}$ \\ ${ }^{1}$ Assistant professor, Dept. of Electrical Engineering, MATS University, Raipur, \\ Chhattisgarh, India \\ ${ }^{2}$ Assistant professor, Dept. of Electronics Engineering, G. H. Raisoni College of \\ Engineering, Nagpur, Maharashtra, India
}

\begin{abstract}
An Inertial Navigation System (INS) can easily track position, velocity and orientation of any moving vehicle. Generally, deterministic errors are present in an uncalibrated Inertial Measurement Unit (IMU) which leads to the requirement of an accurate estimation of navigation solution. These inertial sensors, thus, needs to be calibrated to reduce the error inherent in these systems. By mathematical model of IMU including both accelerometer and gyroscope is utilized for the purpose of error calibration. Particle Swarm Optimization (PSO) and Human Opinion Dynamics (HOD) Optimization based calibration techniques have used to obtain error parameters such as bias, scale factor and misalignment errors.
\end{abstract}

\section{KEYWORDS}

PSO Algorithm, Inertial Measurement Unit, Calibration, HOD Algorithm

\section{INTRODUCTION}

Today Inertial Navigation System (INS) is used in missile guidance, space navigation, marine navigation and navigation sensor in cellular mobile phones. Inertial sensor consists of accelerometers and gyroscopes for three dimension linear and angular motion, respectively. Initially, when inertial sensor was developed, it was very costly and large in size, but after many improvements now inertial sensor is available in solid state chip and cheaper. To overcome the limitations of Global Positioning System (GPS) and for high quality of navigation INS is being used. A tri-axial Inertial Measurement Unit (IMU), includes a traid of accelerometers and gyroscopes. Accelerometers are mounted to estimate the velocity and position (linear motion) of the aircraft or vehicle and gyroscopes are mounted to keep the orientation in the space (to measure angular motion) [1]. By using accelerometers and gyroscopes the location of any aircraft or vehicle can be tracked easily. But, in accelerometers and gyroscopes some errors are present which is inherent to the IMU.

To use Inertial Navigation System properly, calibration is required. Because, IMUs are typically not compensated initially, present errors increase gradually with time and distance. Errors in inertial sensor can be classified into two main parts [2]: deterministic or systematic and random 
or stochastic errors. In recent past, it is studied that, for INS calibration different types of methods are used such as six-position method, improved six-position method and multi-position method. To calibrate all deterministic errors which are available in INS, these twelve errors are such as three biases, three scale factors and six misalignments [3]. After many improvements, low cost INS can be calibrated easily without external equipment [4].

In this work of low cost INS calibration, two sophistic optimization techniques are being used such as Particle Swarm Optimization (PSO) and Human Opinion Dynamics (HOD). PSO is inspired from natural behavior of birds or animals and HOD is inspired from opinions of human. It may take few minutes to give output during optimization of given fitness function. HOD is inspired from social behavior and social influence, which has observed from human's social life. After completion of these optimization techniques twelve unknown parameters can be evaluated as a result, which are required for calibrated IMU.

\section{Inertial Measurement Unit (IMU) SEnSor Error Model}

An Inertial Measurement Unit (IMU) is an electronic device that measures velocity, gravitational forces, and angular motion with the help of accelerometers and gyroscopes. An inertial measurement unit works even when GPS (Global Positioning System) receiver is not receiving any signal from the satellite like as inside the tunnels and buildings or in presence of any electronic signals interference. An inertial measurement unit measures linear and angular motion by using accelerometer and gyroscope respectively, which are mounted on an IMU [5]. Inertial navigation sensor contains a tri-axial IMU.

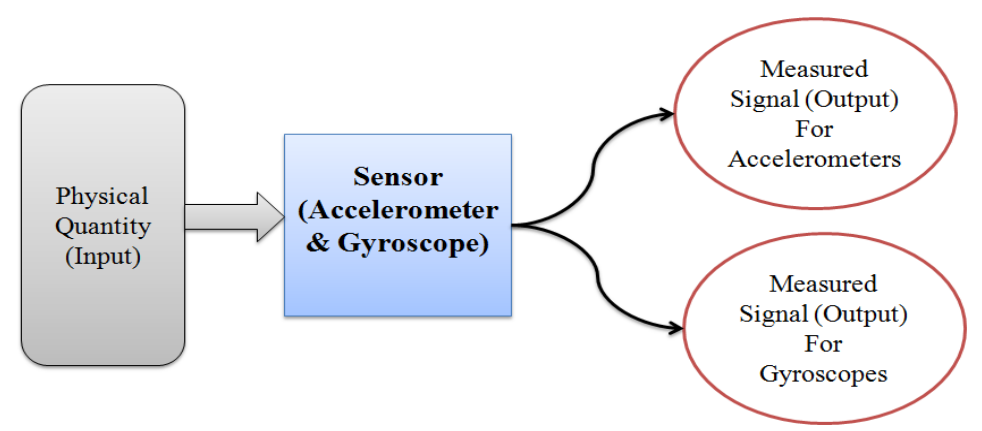

Figure 1: Flow of physical quantity input to measured signals for accelerometers and gyroscopes

It can be seen that, Deterministic and stochastic errors are present in IMU by which getting low quality navigation from the sensor. In this paper deterministic errors are going to be discussed. Deterministic errors include, bias, scale factor, misalignment and non-orthogonality errors for accelerometer and gyroscope respectively as shown in figure 2 . Accelerometer bias can be seen if without linear motion measurable offset has collected and similarly without any angular motion gyroscope bias offset can be observed. Ideally scale factors must be unity, but if the values of scale factors are greater than or less than the unity, scale factor errors are available in accelerometers and gyroscopes respectively. Accelerometer misalignment errors can be seen if all three accelerometers are not aligned properly with the reference frame. Similarly gyroscope misalignment errors also can be detected if all three gyroscopes are not aligned properly with respective axis. 
International Journal Of Instrumentation And Control Systems (IJICS) Vol.7, No.1, January 2017

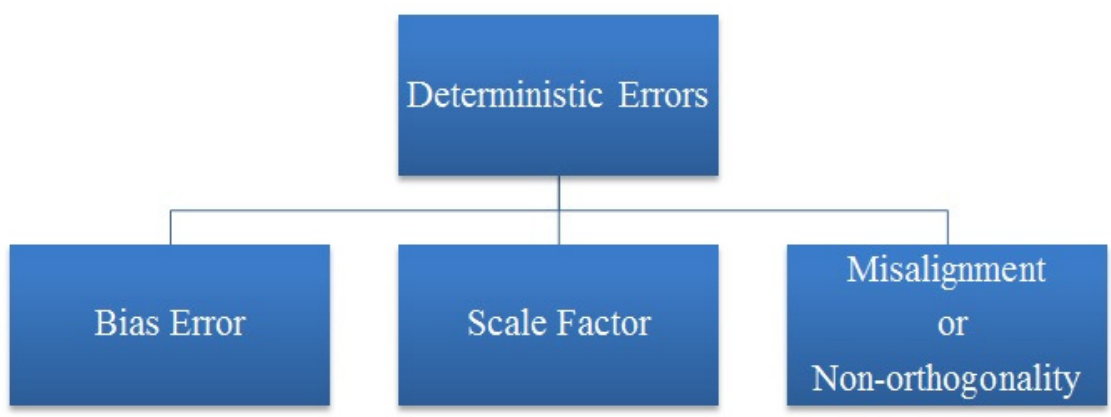

Figure 2: Classification of deterministic or systematic errors for accelerometer and gyroscope

The IMU sensor model represents the measurements from the actual physical quantity to the IMU sensor output as shown in figure 1. If an IMU sensor is given which is calibrated, its bias, scale factor and misalignment or non-orthogonality errors are available for generating a true value from uncalibrated sensor data. Therefore, the accelerometer error model for these deterministic errors is as followed:

$Y_{a}=\left(K_{a}\right)^{-1} * A+B_{a}$

Where,

$K_{a}=S_{a} \times M_{a}$

Equation 2.1 can be rearranged in the form:

$A=\left(K_{a}\right) * Y_{a}-B_{a}$

Where

$Y_{a}=$ raw measured output vector $(3 \times 1)$ from sensor for accelerometer $=\left[\begin{array}{lll}l_{a x} & l_{a y} & l_{a z}\end{array}\right]^{T}$

$S_{a}=$ scale factor vector $(3 \times 3)$

$M_{a}=$ misalignment vector $(3 \times 3)$

$B_{a}=$ bias vector $(3 \times 1)=\left[\begin{array}{l}b_{a x} \\ b_{a y} \\ b_{a z}\end{array}\right]$

$A=$ true value of sensor measurement vector $(3 \times 1)=\left[\begin{array}{lll}g_{a x} & g_{a y} & g_{a z}\end{array}\right]^{T}$

Here scale factor can be written in matrix form in their respective axis $x, y$ and $z$ :

$S_{a}=\left[\begin{array}{ccc}S_{a x} & 0 & 0 \\ 0 & S_{a y} & 0 \\ 0 & 0 & S_{a z}\end{array}\right]$ 
Misalignment error can be represented their respective axis $x, y$ and $z$ in matrix form:

$M_{a}=\left[\begin{array}{ccc}1 & \alpha_{a y z} & \alpha_{a z y} \\ \alpha_{x a z} & 1 & \alpha_{a z x} \\ \alpha_{a x y} & \alpha_{a y x} & 1\end{array}\right]$

$\alpha_{i j}$ are present misalignments in the sensor of the $i^{t h}$ sensitive axis of acceleration around the $j^{t h}$ sensitive axis of the sensor [16].

To estimate the fitness function for accelerometer [7]:

$$
\begin{aligned}
& g_{a x}=S_{a x}\left(l_{a x}-b_{a x}\right)+\alpha_{a y z} S_{a x}\left(l_{a y}-b_{a y}\right)+\alpha_{a z y} S_{a x}\left(l_{a z}-b_{a z}\right) \\
& g_{a y}=\alpha_{a x z} S_{a y}\left(l_{a x}-b_{a x}\right)+S_{a y}\left(l_{a y}-b_{a y}\right)+\alpha_{a z x} S_{a y}\left(l_{a z}-b_{a z}\right) \\
& g_{a x}=\alpha_{a x y} S_{a z}\left(l_{a x}-b_{a x}\right)+\alpha_{a y x} S_{a z}\left(l_{a y}-b_{a y}\right)+S_{a z}\left(l_{a z}-b_{a z}\right) \\
& f_{a}=\sqrt{\left|\left(g_{a x}\right)^{2}+\left(g_{a y}\right)^{2}+\left(g_{a z}\right)^{2}-\left(g_{e}\right)^{2}\right|}
\end{aligned}
$$

In this way from sensor error model deterministic errors have 12 parameters: three for biases, three for scale factors and six for misalignments or orthogonality errors. These all 12 parameters can be rewritten in single vector form:

$\emptyset_{a=}\left[b_{a x} b_{a y} b_{a z} S_{a x} S_{a y} S_{a z} \alpha_{a y z} \alpha_{a z y} \alpha_{a x z} \alpha_{a z x} \alpha_{a x y} \alpha_{a y x}\right]$

For accelerometer, after calculating $f_{a}$ by Equation 9, deterministic errors can be evaluated. Similarly, fitness function can be estimated for gyroscope:

$$
\begin{aligned}
& g_{\omega x}=S_{\omega x}\left(l_{\omega x}-b_{\omega x}\right)+\alpha_{\omega y z} S_{\omega x}\left(l_{\omega y}-b_{\omega y}\right)+\alpha_{\omega z y} S_{\omega x}\left(l_{\omega z}-b_{\omega z}\right) \\
& g_{\omega y}=\alpha_{\omega x z} S_{\omega y}\left(l_{\omega x}-b_{\omega x}\right)+S_{\omega y}\left(l_{\omega y}-b_{\omega y}\right)+\alpha_{\omega z x} S_{\omega y}\left(l_{\omega z}-b_{\omega z}\right) \\
& g_{\omega x}=\alpha_{\omega x y} S_{\omega z}\left(l_{\omega x}-b_{\omega x}\right)+\alpha_{\omega y x} S_{\omega z}\left(l_{\omega y}-b_{\omega y}\right)+S_{\omega z}\left(l_{\omega z}-b_{\omega z}\right) \\
& f_{\omega}=\sqrt{\left|\left(g_{\omega x}\right)^{2}+\left(g_{\omega y}\right)^{2}+\left(g_{\omega z}\right)^{2}-\left(g_{\omega}\right)^{2}\right|}
\end{aligned}
$$

Where, $g_{\omega}=7.292115 \times 10^{-5} \mathrm{rad} / \mathrm{sec}, b_{\omega x}, b_{\omega y}$ and $b_{\omega z}$ are biases in $x, y$ and $z$ respective axis, $S_{\omega x}, S_{\omega y}$ and $S_{\omega z}$ are scale factors in their sensitive axis $x, y$ and $z$ axis. $\alpha_{\omega y z}, \alpha_{\omega z y}, \alpha_{\omega x z}, \alpha_{\omega z x}, \alpha_{\omega x y}$ and $\alpha_{\omega y x}$ are misalignment errors of gyroscope.

These errors can be arranged in an array form:

$$
\emptyset_{\omega}=\left[b_{\omega x} b_{\omega y} b_{\omega z} S_{\omega x} S_{\omega y} S_{\omega z} \alpha_{\omega y z} \alpha_{\omega z y} \alpha_{\omega x z} \alpha_{\omega z x} \alpha_{\omega x y} \alpha_{\omega y x}\right]
$$

After estimation of fitness function for given range of parameters of accelerometers and gyroscopes, optimization techniques can be used for optimization. 
International Journal Of Instrumentation And Control Systems (IJICS) Vol.7, No.1, January 2017

\section{OPTIMIZATION TECHNIQUES}

Optimization Technique is an act to find the best fitting parameters for a given minimization argument. Optimization is a mathematical form which is concerned with finding the maxima or minima of the fitness functions. Generally fitness functions can be subjected to constraint, which needs to be provided carefully depending on the knowledge of the range of values that the unknown variables may have. There are many optimization techniques which are developed to minimize the cost function or fitness function. Particle Swarm Optimization and Human Opinion Dynamics technique has explained in section 3.1 and 3.2 respectively.

\subsection{Particle Swarm Optimization (Pso) Method}

Particle Swarm Optimization (PSO) was originally published by Kennedy and Eberhart (1995) [6]. Basically, PSO is inspired by co-operative behavior observation of social animals and birds in the nature. Compared with other available different type of optimization techniques, PSO is easier to implement and to get optimized solution.

In PSO optimization technique, suppose numbers of particles are available here with in the provided rages. Each particle has four attributes including their current position vector $\left(x_{i}\right)$, their local best position $\left(l_{\text {best }}\right)$, the velocity vector of swarm particles $\left(v_{i}\right)$ and best global position $\left(g_{\text {best }}\right)$. Now the position and velocity of the swarm particles can be updated by [18]:

$v_{i}(t+1)=\omega \times v_{i}(t)+c_{1} \times r_{i 1} \times\left(l_{\text {best } i}(t)-x_{i}(t)\right)+c_{2} \times r_{i 2} \times\left(g_{\text {best }}(t)-x_{i}(t)\right)$

$x_{i}(t+1)=x_{i}(t)+v_{i}(t) \times \Delta t$

Where, $i=1,2,3 \ldots \mathrm{N}$

$\omega=$ inertial weight factor of swarm particles

$c_{1}, c_{2}=$ acceleration coefficients

$r_{i 1}, r_{i 2}=$ random numbers uniformly distributed with in $[0,1]$ range

$\Delta t=$ interval discrete time (set to 1 )

Here $\omega$ can be calculated from:

$\omega=\omega_{\max }-\frac{\left(\omega_{\max }-\omega_{\min }\right) \times \text { current iteration }}{\text { Maximum number of iteration }}$

In Equation 18, $\omega_{\max }=0.9, \omega_{\min }=0.4$, where, $\omega$ can be vary with in the given range and it can be seen that $\omega$ is decreasing with the iteration and acceleration factor $c_{1}=c_{2}=2$ [17]. Local best position of particle swarms can be updated by [19]:

$$
\begin{aligned}
l_{\text {best } i}(t+1) & =l_{\text {best } i}(t) ; \text { if } f_{a}\left(x_{i}(t)\right)>f_{a}\left(l_{\text {best } i}(t)\right) \\
& =x_{i}(t+1) ; \text { if } f_{a}\left(x_{i}(t)\right) \leq f_{a}\left(l_{\text {best } i}(t)\right)
\end{aligned}
$$

To evaluate $g_{\text {best }}(t)$ : 
$g_{\text {best }}(t+1)$ will be one of the appropriate $l_{\text {best } i}(t+1)$ which will evaluate minimum fitness function [19].

$g_{\text {best }}(t+1)=\arg \min f_{a}\left(l_{\text {best } i}(t+1)\right) \forall i \in N$

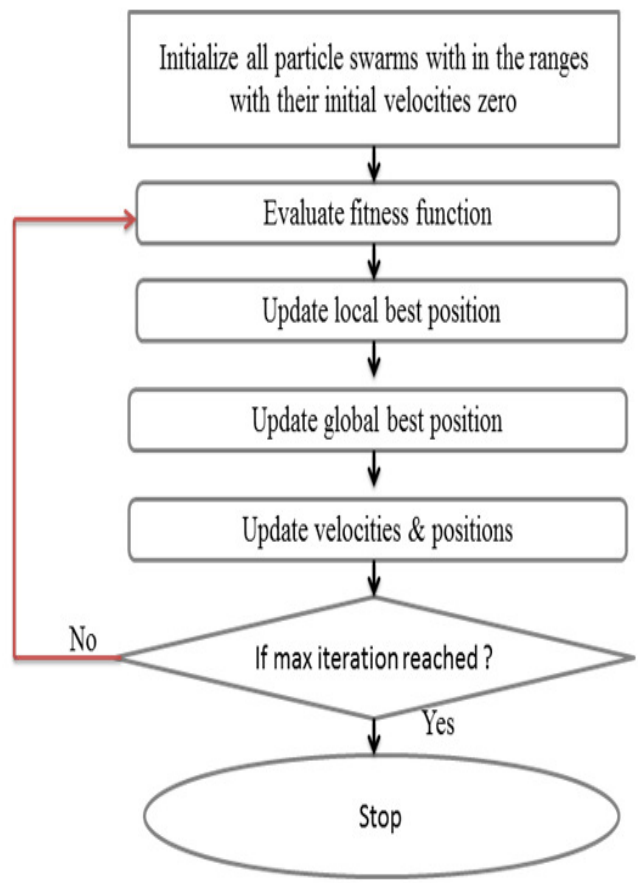

Figure 3: The Particle Swarm Optimization algorithm flow chart

In this way, by using PSO optimization method IMU (accelerometer and gyroscope) can be calibrated by using fitness function from Equation9 and 14, basic PSO optimization technique can be applied [8]. This iterative process will follow the flow chart of Particle Swarm Optimization (PSO) algorithm is mentioned in figure 3.

\subsection{Human OPINION DYNAMics OPTIMIZATION METHOD}

Human opinions are very important area to get some desired conclusion in social life. Human opinion dynamics lead to decision making ability in social life. This human opinion concept can be used to solve complex optimization problem. In real life, it can be seen that, suppose some human opinions are available and their opinions are influenced with each other than, if any human opinion is providing most influence to other then that opinion will be considered as most preferable and given a highest rank over all human opinions. Similarly, if any other human opinion is influencing lesser then last ranker then it will get lesser rank. In this way, all human opinion will be sorted in ascending order.

Continuous opinion dynamics optimizer (CODO) can be used to solve complex mathematical problem, where the basic roots of this algorithm are social structure, opinion space, social influence and updating rule. Social structure has very important role in this algorithm, because it 
governs the interaction between two different individual human opinions and among all individuals.

The second basic important thing is opinion space. In social physics terms, opinions are basically of two types: discrete and continuous. Discrete opinions can be values such as $\{0,1\}$ or $\{-1,1\}$ and continuous could be any real value. Here, continuous opinion will be preferred. $O_{i}(t)$ is opinion vector at a time $t$ where $i=1,2,3 \ldots \mathrm{N}$. The opinion vectors should be uniformly distributed [9].

Social influence is third important rudiment of the algorithm. Each opinion should have decision making ability. Where opinions are influenced with each-other directly or indirectly and social influence is the combined effect of these influences. To estimate the social influence, two factors are responsible, i.e., Social Ranking $(S R)$ of individuals and distance between two different individuals $(d)$. $S R$ can be calculated by their fitness evaluation in ascending order.

The social influence $w_{i j}(t)$ at a time $\mathrm{t}$ of individual $j$ on individual $i$ can be:

$w_{i j}(t)=\frac{S R_{j}(t)}{d_{i j}(t)}$

Where $d_{i j}(t)$ is Euclidean distance between individual $j$ and $i$. And finally updating rule is one of the important aspects to be considered in this algorithm. For updating the opinions, various strategies are adopted as stated in the literatures [10-14]. But, here Durkheimian opinion dynamics has been used for updating rule. The update rule can be:

$$
\Delta O_{i}(t)=\frac{\sum_{j=1}^{N}\left(O_{j}(t)-O_{i}(t)\right) \times w_{i j}(t)}{\sum_{j=1}^{N} w_{i j}(t)}+\xi_{i}(t) ; i \neq j
$$

Where, $O_{j}(t)$ is the neighbors of an individual $i .(j=1,2,3 \ldots \mathrm{N})$ and $\xi_{i}(t)$ is normally distributed random noise with mean zero and standard deviation $\sigma_{i}(t)$ at a time $t$.

Where, $S$ is the strength of disintegrating force of society $(S=0.0001,0.001,0.01,0.1,1,10,100$ as suitable) and $f_{i j}(t)$ is the modulus of difference between fitness of the individual $j$ and $i$ respectively at a time $t$. If $\sigma_{i}(t)$ is higher, higher is the tendency of and individual towards individualization. It means if random noise is generated in Equation 22, then by using this algorithm it will directly converge and get wrong converging point hence, $\xi_{i}(t)$ (normally distributed random noise) has very important role for updating rule. So, $\xi_{i}(t)$ is referred as adaptive noise [15]. In this way by using these equations $(21,22$ and 23) optimized solution can be determined as shown in table 1 .

$\sigma_{i}(t)=S \times \sum_{j=1}^{N} e^{-f_{i j}(t)}$ 
Table 1: Flow of algorithm for human opinion dynamics

$\begin{aligned} & \text { Initialize the opinions for all dimension within the given range and } \\ & \text { here number of opinions will be } \mathbf{N}\end{aligned}$
iteration $=0$
WHILE (iteration $\leq$ maximum iteration) DO
Estimate the fitness of their individual opinion
calculate the social ranking by shorting them in ascending order
iteration = iteration +1
FOR each individual and each dimension
calculate $w_{i j}(t)$ between $\mathrm{j}$ individual i on
Apply updating rule
Run until iteration reaches up to maximum iteration

\section{Results}

For the tri-axial accelerometer, IMU can be rotated in three-dimensional space and there is no need to put the IMU at a desired angle or rotation at particular angular speed. By rotating IMU in space, raw data can be collected which are affected by deterministic errors such as biases, scale factors and misalignment or non-orthogonalities for one minute and fourty seconds. Model parameters of accelerometer and gyroscope have mentioned below in table 2 and 3 of PSO optimization and HOD optimization techniques respectively. In the below tables $\alpha_{\omega y z}, \alpha_{\omega z y}$, $\alpha_{\omega x z}, \alpha_{\omega z x}, \alpha_{\omega x y}$ and $\alpha_{\omega y x}$ are misalignments $b_{x}, b_{y}$ and $b_{z}$ are bias errors, $S_{x}, S_{y}$ and $S_{z}$ are respectively scale factors for accelerometer and gyroscope.

\subsection{TEST ENVIRONMENT}

In laboratory Xsens MTi-G-700 INS is available. MTi-G-700 INS includes an onboard GPS receiver. The MTi-G-700 INS is not only to provide output and GPS-enhanced three dimension orientation but also provides AHRS (Arithmetic Heading and Reference System)-augmented position and velocity in three dimension space. Xsens MTi-G-700 INS is very small in size, low weight, low cost, very flexible with a wide range of interfacing options. Xsens MTi-G-700 INS gives output even if sensor is rotating in three dimensional. By rotating Xsens MTi-G-700 INS in three dimension space, uncalibrated data of the sensor can be measured and collected in xlxs format. These measured data can be direct used in MATLAB as input (uncalibrated data). 
International Journal Of Instrumentation And Control Systems (IJICS) Vol.7, No.1, January 2017

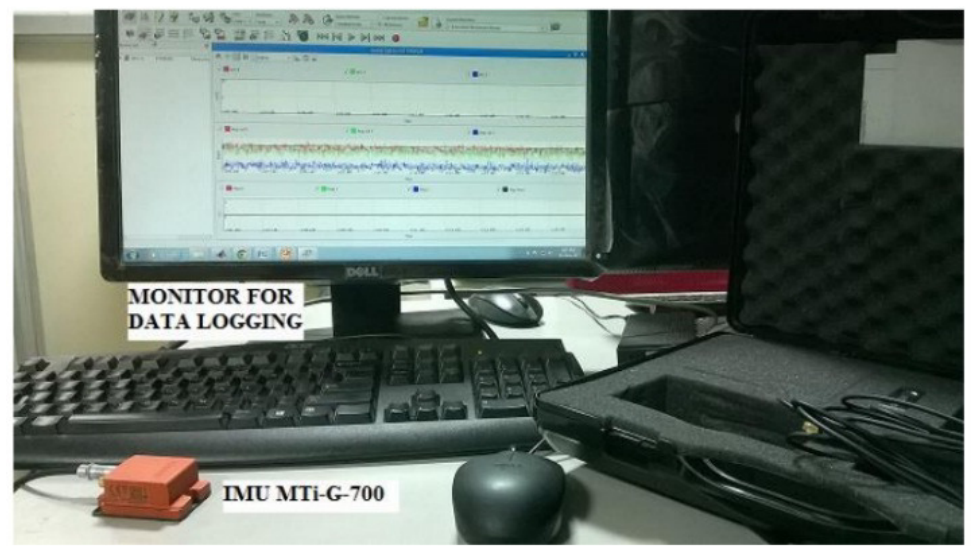

Figure 4: Experimental arrangement of Xsens MTi-G-700

MTi-G-700 INS is directly connected to computer via USB cable. MT manager software is used to collect the data and can be displayed in computer's screen in real time. The motion of the sensor can be linear and angular. The INS output data is decoded at $100 \mathrm{~Hz}$. The output of the accelerometer will be in $\mathrm{m} / \mathrm{s}^{2}$ and output of gyroscope will be in $\mathrm{rad} / \mathrm{sec}$.

The mathematical model is developed as a fitness function from the error model of INS and applied to standard Particle Swarm Optimization and Human Opinion Dynamics optimization techniques, under MATLAB R2013a environment. According to the proposed methodology, error model can be calibrated by these two optimization techniques independently to estimate the unknown parameters.

\subsection{Results For Calibration Using Pso Optimization}

After evaluation of error model, PSO optimization technique can be applied on fitness function of Equation 9 and 14 for accelerometer and gyroscope respectively. Evaluated errors for accelerometer and gyroscope by PSO has shown below in table 2 and these error parameters can be arranged in form of array in Equation 10 and 15 for accelerometer and gyroscope respectively.

Table 2: Errors for accelerometer and gyroscope by PSO optimization technique

\begin{tabular}{|c|c|c|}
\hline Error Parameters & Accelerometer & Gyroscope \\
\hline$b_{x}$ & 32116.3048 & 32178.8073 \\
\hline$b_{y}$ & 31393.2765 & 33282.2932 \\
\hline$b_{z}$ & 31411.0244 & 33680.8198 \\
\hline$S_{x}$ & 0.00627 & 0.000204 \\
\hline$S_{y}$ & 0.00636 & 0.000449 \\
\hline$S_{z}$ & 0.00922 & 0.000215 \\
\hline$\alpha_{y z}$ & 0.0176 & 0.0025 \\
\hline$\alpha_{z y}$ & 0.0031 & -0.0281 \\
\hline$\alpha_{x z}$ & 0.0008 & 0.0047 \\
\hline$\alpha_{z x}$ & 0.0225 & -0.0123 \\
\hline$\alpha_{x y}$ & -0.0018 & -0.0288 \\
\hline$\alpha_{y x}$ & 0.0266 & -0.0034 \\
\hline
\end{tabular}




\subsection{Results For CAlibration USING Hod OPTIMization}

Similarly, Human Opinion Dynamics algorithm can be applied to given fitness function of Equation 9 and 14 of IMU which includes all deterministic errors such as biases, scale factors and misalignment in respective sensitive axes. After applying HOD algorithm, deterministic errors can be obtained for accelerometer and gyroscope respectively. In this way, results for a set of sequence of HOD optimization technique can be obtained by collected raw data of IMU which are influenced by deterministic errors for accelerometer and gyroscope.

Table 3: Errors for accelerometer and gyroscope by HOD algorithm

\begin{tabular}{|c|c|c|}
\hline Model parameters & Accelerometer & Gyroscope \\
\hline$b_{x}$ & 32814.6839 & 32182.3256 \\
\hline$b_{y}$ & 32873.7602 & 31401.4845 \\
\hline$b_{z}$ & 32229.5274 & 32319.6946 \\
\hline$S_{x}$ & 0.0071 & 0.0002 \\
\hline$S_{y}$ & 0.0076 & 0.0003 \\
\hline$S_{z}$ & 0.0056 & 0.0002 \\
\hline$\alpha_{y z}$ & 0.0295 & -0.0264 \\
\hline$\alpha_{z y}$ & -0.0285 & -0.0015 \\
\hline$\alpha_{x z}$ & -0.0047 & -0.0235 \\
\hline$\alpha_{z x}$ & -0.0097 & -0.0237 \\
\hline$\alpha_{x y}$ & -0.0007 & -0.0072 \\
\hline$\alpha_{y x}$ & -0.0087 & -0.0197 \\
\hline
\end{tabular}

It can be seen that twelve error parameters have shown in table 2 and 3 for accelerometers and gyroscopes by using PSO and HOD optimization techniques. These twelve parameters can be graphically represented for accelerometers of PSO and HOD optimized values which includes bias, scale factor and misalignment error parameters as shown in figure 5 . Bias errors are in $\mathrm{m} / \mathrm{s}^{2}$, scale factor are unit less and misalignments are $i^{\circ}$ degree in figure 5. Similarly graph representation is shown in figure 6 for gyroscope where, bias errors are in $\mathrm{rad} / \mathrm{sec}$, scale factor are unit less and misalignments are in ${ }^{\circ}$ degree.

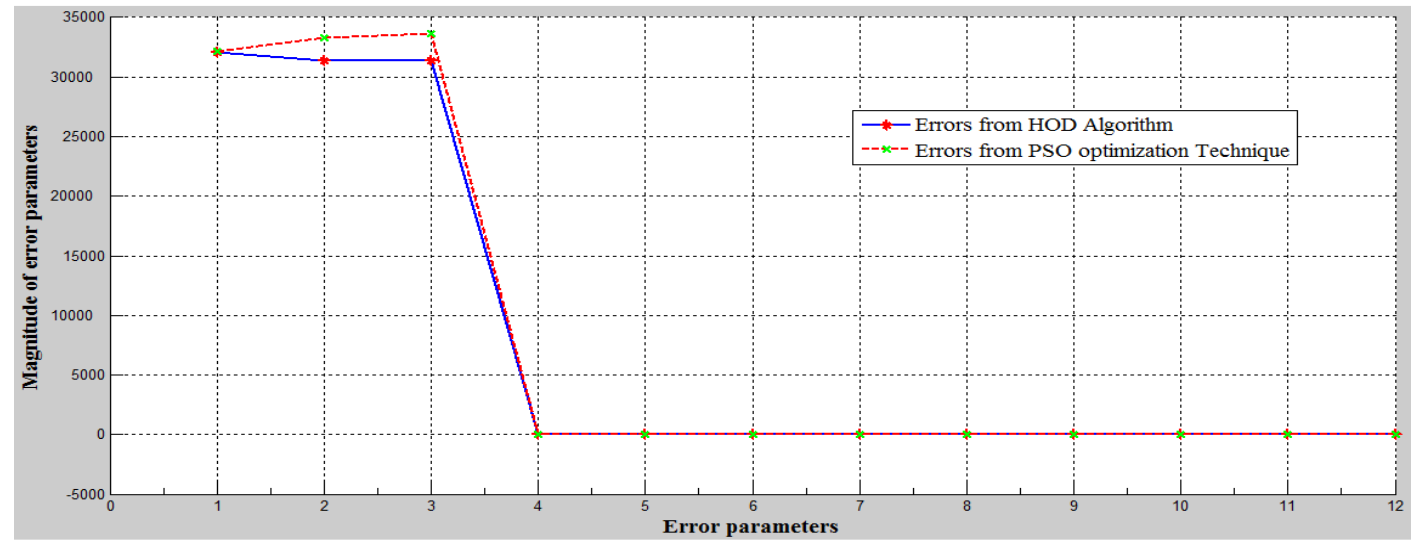

Figure 5: Graph representation of PSO and HOD optimization techniques for accelerometer in respective axis 


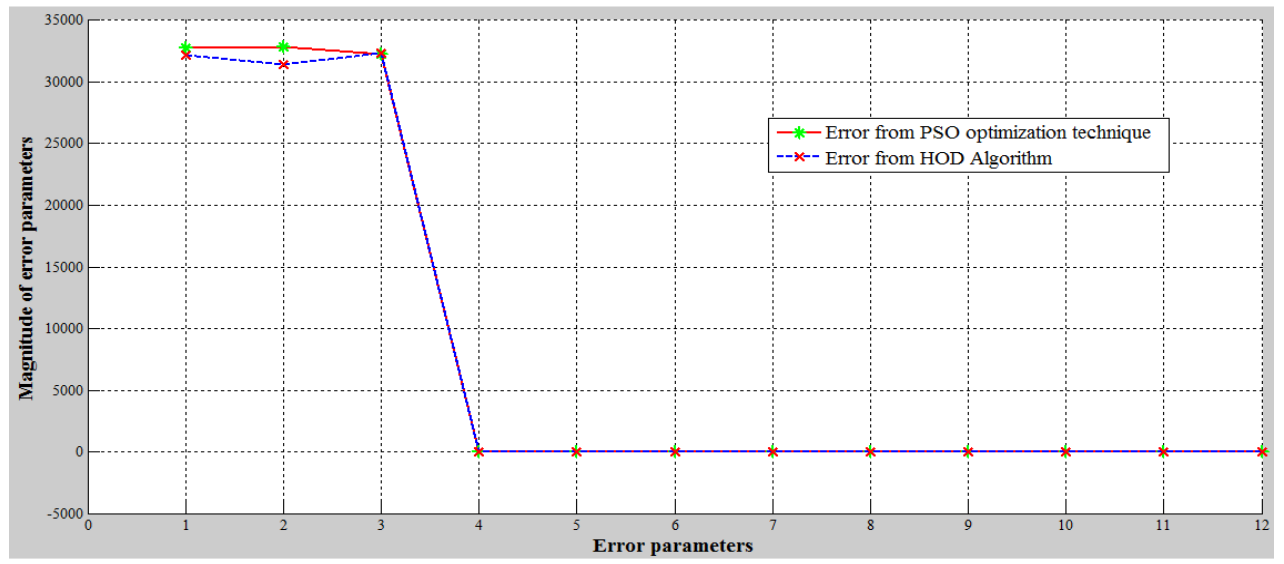

Figure 6: Graph representation of PSO and HOD optimization techniques for gyroscope in respective axis

\section{CONCLUSION}

For calibration purpose, two different and unique methods have been adopted. Low cost MEMS sensors are having large errors compared to higher grade inertial sensors. These deterministic errors must be calibrated to get acceptable navigation results. This study attempted to test the performance of the PSO optimization and Human Opinion Dynamics optimization techniques for IMU calibration. First, Particle Swarm Optimization (PSO) algorithm is applied for calibration purpose and getting all deterministic error parameters and on the other side Human Opinion Dynamics optimization algorithm can be applied on error model of IMU for calibration.

From results, it can be seen that, Particle Swarm Optimization and Human Opinion Dynamics optimization techniques are giving the satisfactory result. However, since this technique does not require any specific set of orientation sequences to be managed, it avoids any costly equipment usage and is to be evolved further.

\section{FUTURE WORKS}

In future, it is aimed to study stochastic error modeling is to be also studied along with the deterministic error. Furthermore, it is also desired to study few more optimization techniques other than PSO and HOD which can be applied here in this scenario. And, lastly it is also aimed in future to combine stochastic and deterministic error modeling in one framework.

\section{REFERENCES}

[1] Unsal, Derya, and Kerim Demirbas. "Estimation of deterministic and stochastic IMU error parameters." Position Location and Navigation Symposium (PLANS), 2012 IEEE/ION. IEEE, 2012.

[2] Nassar, Sameh. Improving the inertial navigation system (INS) error model for INS and INS/DGPS applications. National Library of Canada= Bibliothèque nationale du Canada, 2005.

[3] Tee, Kian Sek, et al. "Triaxial accelerometer static calibration." (2011).

[4] Fong, W. T., S. K. Ong, and A. Y. C. Nee. "Methods for in-field user calibration of an inertial measurement unit without external equipment." Measurement Science and Technology 19.8 (2008): 085202. 
International Journal Of Instrumentation And Control Systems (IJICS) Vol.7, No.1, January 2017

[5] Naranjo, Claudia C. Meruane. "Analysis and modeling of MEMS based inertial sensors." School of Electrical Engineering, Kungliga Tekniska Hgskolan, Stockholm (2008).

[6] Eberhart, Russ C., and James Kennedy. "A new optimizer using particle swarm theory." Proceedings of the sixth international symposium on micro machine and human science. Vol. 1. 1995.

[7] Zhang, Liguo, Wenchao Li, and Huilian Liu. "Accelerometer Static Calibration based on the PSO algorithm." 2nd International Conference on Electronic \& Mechanical Engineering and Information Technology. Atlantis Press, 2012.

[8] Bai, Qinghai. "Analysis of particle swarm optimization algorithm." Computer and information science 3.1 (2010): 180.

[9] Kaur, Rishemjit, et al. "Human opinion dynamics: An inspiration to solve complex optimization problems." Scientific reports 3 (2013).

[10] Nowak, Andrzej, Jacek Szamrej, and Bibb Latané. "From private attitude to public opinion: A dynamic theory of social impact." Psychological Review 97.3 (1990): 362.

[11] Deffuant, Guillaume, et al. "Mixing beliefs among interacting agents." Advances in Complex Systems 3.01 n04 (2000): 87-98.

[12] Hegselmann, Rainer, and Ulrich Krause. "Opinion dynamics and bounded confidence models, analysis, and simulation." Journal of Artificial Societies and Social Simulation 5.3 (2002).

[13] Sznajd-Weron, Katarzyna. "Sznajd model and its applications." arXiv preprint physics/0503239 (2005).

[14] Mäs, Michael, Andreas Flache, and Dirk Helbing. "Individualization as driving force of clustering phenomena in humans." PLoS Comput Biol 6.10 (2010): e1000959.

[15] Panahandeh, Ghazaleh, Isaac Skog, and Magnus Jansson. "Calibration of the accelerometer triad of an inertial measurement unit, maximum likelihood estimation and Cramer-Rao bound." Indoor Positioning and Indoor Navigation (IPIN), 2010 International Conference on. IEEE, 2010.

[16] Cai, Qingzhong, et al. "Accelerometer calibration with nonlinear scale factor based on multi-position observation." Measurement Science and Technology 24.10 (2013): 105002.

[17] Mahapatra, Prasant Kumar, et al. "Particle swarm optimization (PSO) based tool position error optimization." International Journal of Computer Applications 72.23 (2013).

[18] Zhi-Jie, L. I., et al. "An improved particle swarm algorithm for search optimization." 2009 WRI Global Congress on Intelligent Systems. Vol. 1. IEEE, 2009.

[19] Tan, Chin-Woo, et al. "Design of gyroscope-free navigation systems." Intelligent Transportation Systems, 2001. Proceedings. 2001 IEEE. IEEE, 2001.

[20] Kaplan, Elliott, and Christopher Hegarty. Understanding GPS: principles and applications. Artech house, 2005.

[21] Savage, Paul G. Strapdown analytics. Vol. 2. Maple Plain, MN: Strapdown Associates, 2000.Maybeck, Peter S. Stochastic models, estimation, and control. Vol. 3. Academic press, 1982.

[22] Godha, Saurabh. Performance evaluation of low cost MEMS-based IMU integrated with GPS for land vehicle navigation application. Library and Archives Canada= Bibliothèque et Archives Canada, 2006.

[23] Flenniken, W., J. Wall, and D. Bevly. "Characterization of various IMU error sources and the effect on navigation performance." ION GNSS. 2005.

[24] Grewal, Mohinder S., Lawrence R. Weill, and Angus P. Andrews. Global positioning systems, inertial navigation, and integration. John Wiley \& Sons, 2007.

[25] Chatfield, Averil B. Fundamentals of high accuracy inertial navigation. Vol. 174. Aiaa, 1997.

[26] Titterton, David, and John L. Weston. Strapdown inertial navigation technology. Vol. 17. IET, 2004.

[27] Aggarwal, P., et al. "A standard testing and calibration procedure for low cost MEMS inertial sensors and units." Journal of navigation 61.02 (2008): 323-336.

[28] Artese, G., and A. Trecroci. "Calibration of a low cost MEMS INS sensor for an integrated navigation system." Int. Arch. Photogramm. Remote Sens. Spatial Inf. Sci (2008): 877-882.

[29] Cheuk, Chi Ming, et al. "Automatic calibration for inertial measurement unit." Control Automation Robotics \& Vision (ICARCV), 2012 12th International Conference on. IEEE, 2012.

[30] Skog, Isaac, and Peter Händel. "Calibration of a MEMS inertial measurement unit." XVII IMEKO World Congress. 2006. 
International Journal Of Instrumentation And Control Systems (IJICS) Vol.7, No.1, January 2017

Vikas Kumar Sinha received the B.E. degree in Electronics and Telecommunication from Government Engineering College Bilaspur Chhattisgarh India in 2012 and the M.Tech degree from Manipal Institute of Technology, Karnataka, India in 2015.He is currently Assistant Professor in MATS University Raipur, Chhattisgarh, India. He also worked in CSIR-CSIO Chandigarh as a M.Tech Trainee for 10 months. His specific research interests include INS calibration techniques, optimization techniques.

Avinash Maurya received the B.E. degree in Electronics and Telecommunication from Government Engineering College Bilaspur Chhattisgarh India in 2012 and the M.Tech degree from SGGS IE\&T Nanded, Maharastra, India in 2015. He is currently working as Assistant Professor in G. H. Raisoni College of Engineering, Nagpur, Maharashtra, India. His research interest is fractional order system and optimization.
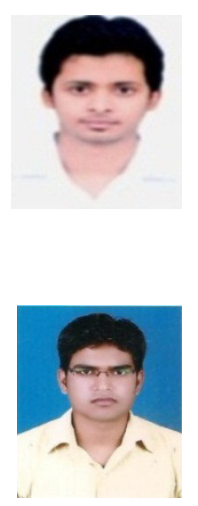